\title{
The impact of bone marrow-derived mesenchymal stem cells on neovascularisation in rats with brain injury
}

\author{
Wei Hu${ }^{1}$, Jian Jiang ${ }^{2}$, Feng Yang ${ }^{2}$, Jun Liu $^{2}$ \\ ${ }^{1}$ Department of Neurosurgery, Enze Medical Centre (Group), Enze Hospital of Taizhou, Taizhou, China, ${ }^{2}$ Department of Neurosurgery, \\ the First People's Hospital of Jiujiang, Jiujiang, China
}

\begin{abstract}
Introduction: The impact of bone marrow-derived mesenchymal stem cells (BM-MSCS) on neovascularisation in rats with craniocerebral injury (Cl) was investigated.

Material and methods: The rate $\mathrm{Cl}$ model was established by the free-fall method, and then rats were randomly divided into a transplanted group $(E G)$ and a control group (CG). Rats in the transplanted group were injected with $B M-M S C s$ in the lateral ventricle, while those in the control group were injected with normal saline. Modified neurological severity scores (mNSSs) were compared. Furthermore, CD34+CD133 double-labelled cells (CD34+CD133+ cells) in the peripheral blood and expression of CD31 and neuron-specific enolase in injured tissues were detected. Results: There were significant intra- and intergroup differences in modified neurological severity scores at different time points. The number of CD34+CD133+ cells in the peripheral blood of CG initially decreased, but it increased later and peaked $6 \mathrm{~h}$ after injury, then gradually decreased and returned to normal $24 \mathrm{~h}$ after injury. Cells in the peripheral blood of EG continued to increase until $24 \mathrm{~h}$ after injury, reaching a number higher than that in $C G(p<0.05)$. The postoperative expression of neuron-specific enolase in $E G$ was higher than that in $C G(p<0.05)$. The positive expression of $C D 31$ was lower in the two groups before surgery, but the expression in EG was higher than that in $C G$ after surgery $(p<0.05)$.

Conclusions: Bone marrow-derived mesenchymal stem cells transplantation can increase the number of endothelial progenitor cells in the peripheral blood of rats with traumatic brain injury and increase the expression of peripheral angiogenetic markers and neuronal markers. The neurological function in EG improved significantly compared to that of $C G$.
\end{abstract}

Key words: bone marrow mesenchymal stem cells, rat, traumatic brain injury, neovascularisation.

\section{Introduction}

The incidence of craniocerebral injury $(\mathrm{Cl})$ is high, and its pathological changes can be divided into primary injury and secondary injury [22,23]; the former is neuronal damage directly caused by external vio- lence, while the latter is caused by a variety of factors, and thus its pathophysiological responses are very complex [7,19], making $\mathrm{Cl}$ one of the main causes of human disability and death. Presently, there are a variety of treatment methods against secondary injury, which can reduce mortality and improve prog- 
nosis, but many patients still suffer from neurological dysfunction and life-long disability, causing a heavy burden to individuals, their families, and societies.

As described above, numerous studies have examined neurological repair. Recent studies have shown that the cerebrovascular system that remains stable after traumatic brain injury can be activated to initiate vessel remodelling $[1,11]$, and the newly formed vasculature can rapidly promote nerve recovery, including nerve regeneration and synaptic regeneration, to promote the recovery of neurological function [13].

Transplanting neural stem cells (NSCs) or bone marrow-derived mesenchymal stem cells (BM-MSCS) can promote angiogenesis after ischaemic brain injury $[5,18,20]$. However, whether endogenous or transplanted NSCs after trauma are involved in neovascularisation after traumatic brain injury remains unclear. In this study, rats with brain injury were divided into an experiment group (EG) and a control group (CG) to analyse and compare their modified neurological severity scores (mNSS), changes in CD34+CD133+ cells in the peripheral blood, and expression of CD31 and neuron-specific enolase (NSE) in injured tissues, to analyse the neovascularisation conditions in peri-injury brain tissue and the impact of BM-MSC transplantation on nerve repair and functional recovery.

\section{Material and methods \\ Animals}

A total of 50 adult male healthy SD rats (120-300 g) were provided by the Experimental Animal Centre of the Department of Medicine, Nanchang University.

This study was carried out in strict accordance with the recommendations in the Guide for the Care and Use of Laboratory Animals of the National Institutes of Health. The animal use protocol was reviewed and approved by the Institutional Animal Care and Use Committee (IACUC) of the First People's Hospital of Jiujiang.

\section{Preparation of rat craniocerebral injury model}

After anaesthetisation by intraperitoneally injecting $10 \%$ chloral hydrate $(1-2 \mathrm{ml} / \mathrm{kg})$, each rat was fixed onto a brain stereotaxic apparatus, sterilised, the scalp was cut open, and one $3-\mathrm{mm}$ round bone window (in diameter) with a point $3 \mathrm{~mm}$ posterior to the anterior fontanelle and $2 \mathrm{~mm}$ left of the sag- ittal line as the centre was drilled. One self-made strike tube was disinfected and then tightly fixed at the edge of the bone window. One 20-g weight was placed $15 \mathrm{~cm}$ down the strike tube to strike the dura mater, striking the right parietal lobe of the dura mater with a striking force of $300 \mathrm{~g} \times \mathrm{cm}$ to induce brain laceration. The bone window was sealed with bone wax and the rat was masked for oxygen supply after suturing the scalp [19].

\section{Isolation, amplification, culture, and identification of bone marrow- derived mesenchymal stem cells}

The tibia and femur were sampled from each rat killed by cervical dislocation (body weight $\sim 120 \mathrm{~g}$ ) and then rinsed with DMEM (Hyclone, Logan, UT, USA). The obtained cell suspension was then centrifuged at $1500 \mathrm{r} / \mathrm{min}$ for $5 \mathrm{~min}$. The cell precipitate was mixed with $10 \%$ foetal bovine serum (SJQ Co., Hangzhou, China)-containing DMEM and cultured at $37^{\circ} \mathrm{C}$ and $5 \% \mathrm{CO}_{2}$; the cells were digested and passaged once every three days (1:2), and P5-generation cells were harvested for analysis.

P3 generation BMSCs were extracted by flow cell phenotype identification and added to three tubes after digestion and suspension. Next, $0.02 \mathrm{ml}$ CD90-fluorescein isothiocyanate (FITC) and CD34-PE (Wuhan Boster Co., Ltd., Wuhan, China) were added to the tubes, and the tubes were incubated in the dark for 30 min. Marked single-cell suspensions were obtained for flow cytometry after washing the cells with PBS.

\section{Transplantation of bone marrow- derived mesenchymal stem cells and grouping}

The rats were divided into the EG and CG groups by using a simplified randomisation method, with 25 rats in each group. Rats in the EG were transplanted with BM-MSCs via intraventricular injection $12 \mathrm{~h}$ after surgery; before transplantation, BrdU (Medchem Express, Monmouth Junction, NJ, USA) (final concentration: $10 \mathrm{mM}$ ) was added to the medium for 2-4 h of co-incubation with the cells. The cells were then digested and adjusted to a density of $1 \times 10^{6}$ cells $/ \mathrm{ml}$, and then each rat in the EG was anesthetised and fixed onto the stereotaxic for injection. Needling point: $\mathrm{AP}=-1 \mathrm{~mm}, \mathrm{ML}=-1.0-1.5 \mathrm{~mm}$, 3.5-4.0 $\mathrm{mm}$ dorsoventral to the skull plane. The needle 
was vertically inserted into the left ventricle and labelled cells $\left(10 \mu \mathrm{l}, 1 \times 10^{4} / \mathrm{rat}\right)$ were slowly injected into the ventricle by one micro-injector. The needle was maintained intracerebrally for $3 \mathrm{~min}$ before suturing the wound. Rats in the CG group were injected with saline intraperitoneally.

\section{Determination of motor function}

Six rats were sampled from each group on day 1 (D1), D3, D7, D14, and D21 after surgery, and motor function was determined by mNSS (Table I) and a double-blind method.

\section{Detection of CD34+CD133+ cells in peripheral blood}

Twelve rats in each group were randomly selected, and $0.60 \mathrm{ml}$ of venous blood was sampled from the venous plexus of the medial canthus on $\mathrm{H} 33 \mathrm{~h}$ after injury), H6, D1, D3, D7, and D14 after surgery using a clean hard glass capillary (1 $\mathrm{mm}$ in diameter), which was then placed into an EDTA-anticoagulated tube to isolate mononuclear cells form the peripheral blood by Ficoll density gradient centrifugation.

Ten microliters of goat anti-rat CD133 monoclonal antibody (primary antibody, Wuhan Boster Co., Ltd.) and $2 \mu \mathrm{l}$ of FITC-labeled rabbit anti-goat IgG monoclonal antibody (secondary antibody, Wuhan Boster Co., Ltd.) were mixed and incubated at $4{ }^{\circ} \mathrm{C}$ in the dark to prepare FITC-labelled CD133 (CD133-FITC); CD34-TRITC (Wuhan Boster Co., Ltd.) was prepared using the same method. The rat mononuclear cells were first added to $100 \mu$ l of $0.50 \%$ foetal bovine serum buffer ( $\mathrm{v} / \mathrm{v})$, mixed evenly, and then $10 \mu \mathrm{l}$ of CD34-TRITC and $10 \mu \mathrm{l}$ of CD133-FITC were mixed, followed by low-speed agitation, full rinsing, 10-min incubation at room temperature in the dark, rinsing,

Table I. Modified rat Neurological Severity Scoring

\begin{tabular}{|c|c|}
\hline Index & Score \\
\hline \multicolumn{2}{|l|}{ Exercise test } \\
\hline Tail lifting & 3 \\
\hline Forelimb flection & 1 \\
\hline Hindlimb flection & 1 \\
\hline Head deflecting from the vertical line within $30 \mathrm{~s}>100$ & 1 \\
\hline Exercises on plane floor (normal = 0 point; maximum = 3 points) & 3 \\
\hline Can walk normally & 0 \\
\hline Cannot walk in straight line & 1 \\
\hline Circling toward the paralytic side & 2 \\
\hline Falling toward the paralytic side & 3 \\
\hline Sensory test & 2 \\
\hline Placement test (vision and touch sensation test) & 1 \\
\hline Proprioception (deep sensation, pressing rat paw against the table edge so as to stimulate the limb muscles) & 1 \\
\hline Balancing beam test (normal = 0 point; maximum = 6 points) & 6 \\
\hline Stabilise the equilibrium status & 0 \\
\hline Tightly seize the edge of balancing beam & 1 \\
\hline Tightly seize the edge of balancing beam, while one limb falls from the balancing beam & 2 \\
\hline $\begin{array}{l}\text { Tightly seize the edge of balancing beam, while two limbs fall from the balancing beam or rotate on the balancing } \\
\text { beam (>60 s) }\end{array}$ & 3 \\
\hline Try to balance on the balancing beam but falls (> $40 \mathrm{~s}$ ) & 4 \\
\hline Try to balance on the balancing beam but falls (> $20 \mathrm{~s}$ ) & 5 \\
\hline Falls while not trying to balance on the balancing beam $(<20 \mathrm{~s})$ & 6 \\
\hline Reflection loss and abnormal motions & 4 \\
\hline Auricle reflex (shaking head when being touched the external acoustic meatus) & 1 \\
\hline Corneal reflex (blinking when being touched the cornea using cotton silk) & 1 \\
\hline Corneal reflex (responses to noises of flipping cardboard) & 1 \\
\hline Depressive, myoclonia, myodystonia & 1 \\
\hline
\end{tabular}


and centrifugation to discard the supernatant and prepare the mononuclear cell suspension. The number of CD34+CD133+ cells in the mononuclear cell suspension was detected by flow cytometry.

\section{Detection of neuron-specific enolase and CD31 in peri-injury brain tissue}

Two rats from each group were randomly selected and killed at different time points (before surgery, H6, D1, D3, D7, D14, and D21 after surgery), followed by $4 \%$ paraformaldehyde perfusion, fixation and dehydration in neutral formaldehyde solution, wax embedding, slicing of the site with obvious injury, haematoxylin and eosin (HE) staining, and detection of CD31 (Wuhan Boster Co., Ltd.) and NSE (Wuhan Boster Co., Ltd.) by the SP immunohistochemical method. Four sites were selected from each slice under a low-magnification microscope $(\times 100)$, and each site was photographed five times using a high-magnification microscope $(\times 400)$. The images were analysed using the Image Pro plus 6.0 colour pathological image analysis system; the mean optical density and relative area (\%) of the positive area of each image were measured, and the product of these two was used to determine the relative content.

\section{Microvessel identification and density peri-injury brain tissue}

Immunohistochemical staining was used to detect the positive expression of CD31 in peri-injury brain tissue before and 3, 7, and 14 days after surgery. Endothelial cells stained as brownish yellow particles were defined as positive. Low-magnification scans of the whole slice were acquired to find the "hotspot" with maximum vascular density and determine the average number of blood ves sels stained from three high magnifications $(\times 400)$ to represent microvessel identification and density (MVD). The microvessel was defined from the separation of endothelial cells and nearby tissue, but not by the vascular lumen or erythrocyte, and excluded lumen with a diameter more than $60 \mu \mathrm{m}$.

\section{BrdU-positive cells in the experimental peri-injury brain tissue group}

Fourteen days after brain injury, the expression of BrdU was detected by immunohistochemistry using the primary antibody anti-mouse monoclonal antibody 5-bromodeoxyuridine. Positive expression appeared as brown particles in the nucleus. The inspected area was defined by low magnification $(\times 100)$, which was the edge of injury grey matter and white matter, while morphological changes were observed at high magnification $(\times 200$ and $\times 400)$.

\section{Statistical analysis}

SPSS 16.0 (SPSS, Inc., Chicago, IL, USA) was used for statistical analysis. The measurement data are expressed as $\bar{x} \pm \mathrm{s}$; intergroup comparison was conducted by the paired $t$ test or independent-sample $t$ test, and intragroup comparison was conducted by analysis of variance (ANOVA), with the test level $\alpha$ as 0.05 .

\section{Results}

\section{Amplification, culture, and identification of bone marrow-derived mesenchymal stem cells}

After seeding, the cells gradually showed flask wall-adherent growth, the number of third-generation BM-MSCs was low, and the cells were scattered and had clustered spindle or triangular shapes. With the rapid proliferation of BMSCs and natural selection of wall-adherent predominance, P3-generation cells were purified to only wall-adherent cells (Fig. 1). The phenotype was tested based on P3 cells by flow cytometry: the expression of CD90 was positive in P3 cells (68.49\%) while the expression of CD34 was negative $(2.85 \%)$, as shown in Figure 2.

\section{CD34+CD133+ cells in peripheral blood}

Mononuclear cells in the peripheral blood were isolated at different time points, and the number of CD34+CD133+ cells in mononuclear cells was detected by flow cytometry. CD34+CD133+ cells in the circulation of peripheral blood were approximately 4052 cells/200,000 mononuclear cells, with the average being 47/200,000 mononuclear cells. The number of CD34+CD133+ cells in the peripheral blood in group CG decreased until $3 \mathrm{~h}$ after injury and then increased to normal. The average number of CD34+CD133+ cells reached the highest level $6 \mathrm{~h}$ after injury, with an average of 66 cells/200,000 mononuclear cells, which gradually decreased and returned to normal $24 \mathrm{~h}$ after injury. The EG group exhibited the same trend; however, the number of CD34+CD133+ cells continued to increase until $24 \mathrm{~h}$ after injury, and 

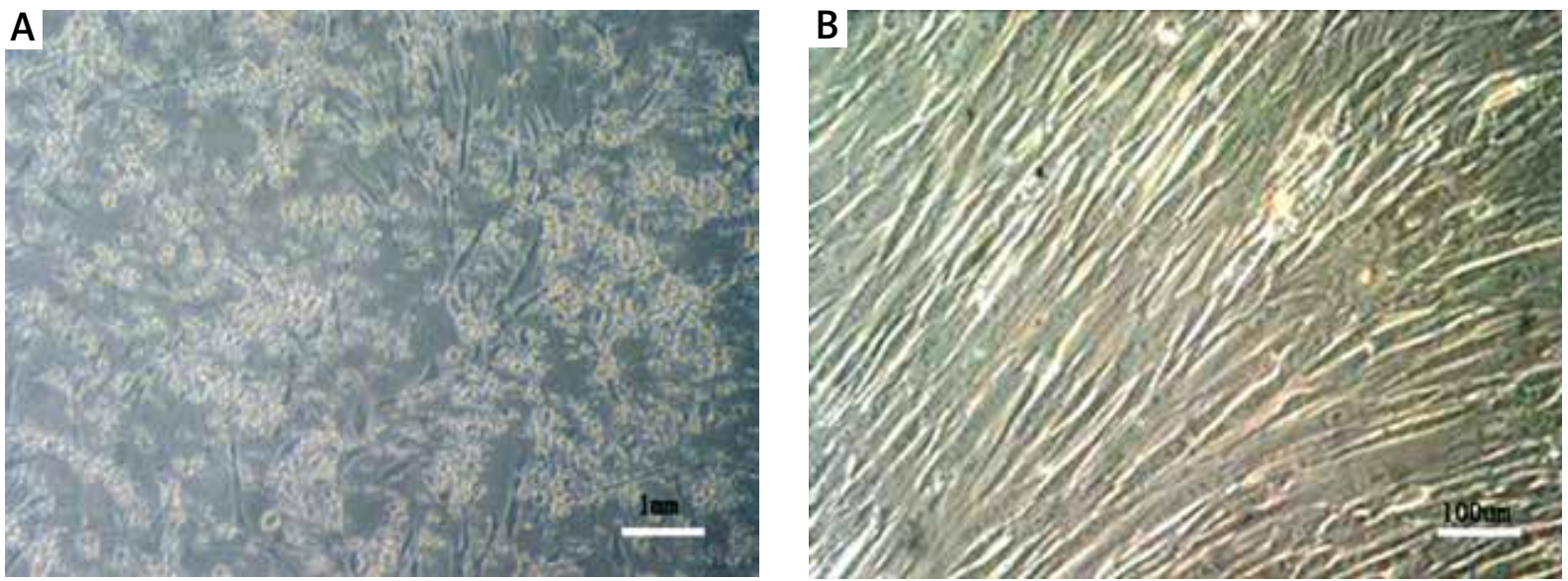

Fig. 1. Amplification and culture of bone marrow-derived mesenchymal stem cells. A) Primary cultured cells on day 3 , a few cells begin adherent, showed short fusiform shape or polygon, and most of the cells suspended and grew colony like (OLYMPUS, $\times 10)$. B) P3 cultured cell morphology, most cells were adherent of spindle shape and long spindle (OLYMPUS, $\times 100)$.

A

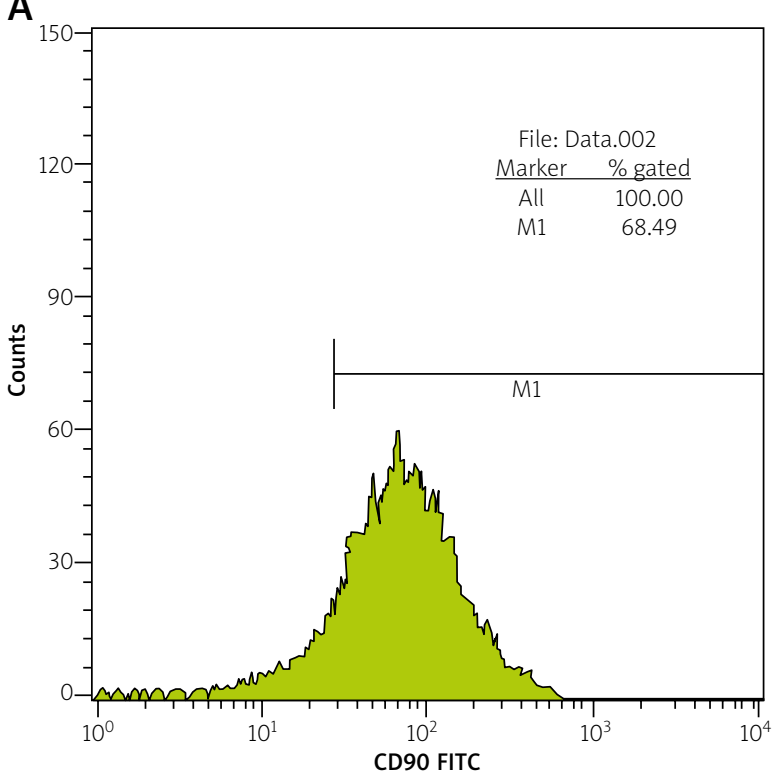

B

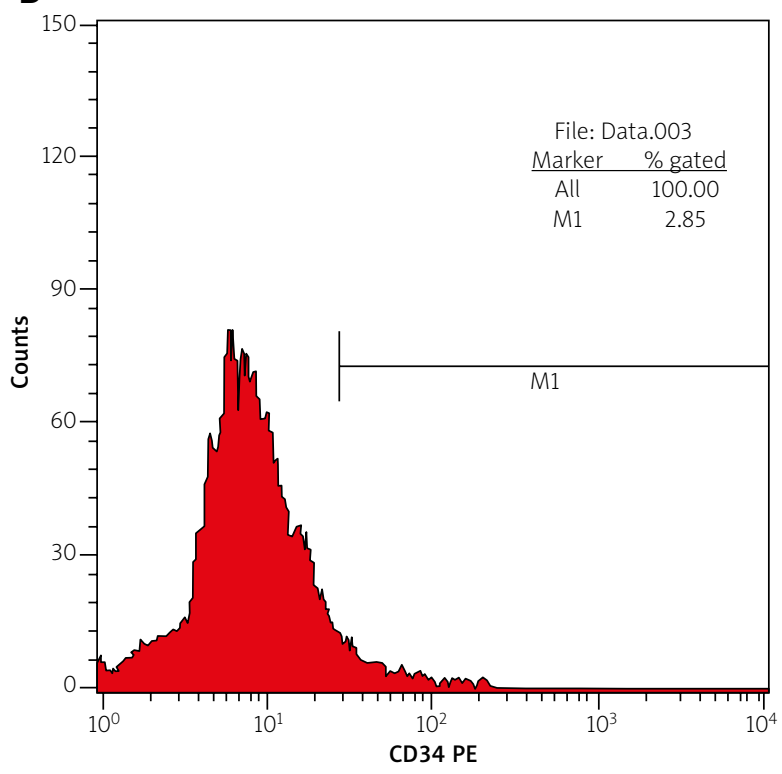

Fig. 2. The expression of CD90 and CD34 of P3-generation cells by flow cytometry. A) The expression of CD90 was positive (68.49\%). B) The expression of CD34 was negative (2.85\%).

the number was significantly higher than that in CG group ( $t$ test, $p<0.05$, Table II; Figs. 3,4 ).

\section{Expressions of neuron-specific enolase and CD31 in peri-injury brain tissue}

The relative contents of NSE and CD31 were detected with the Image pro plus 6.0 colour pathological image analysis system. The results are shown in Figures 3 and 6.
Positive expression of CD31 in the two groups before surgery was low, but began increasing slightly at postoperative $6 \mathrm{~h}$ and 1 day; CD31 was highly expressed on postoperative day 3 , reaching a peak on postoperative D7, and began decreasing on postoperative D14. There were significant differences in the expression of CD31 between the EG and CG groups on postoperative D1, D3, and D7 $(p<0.05)$. 


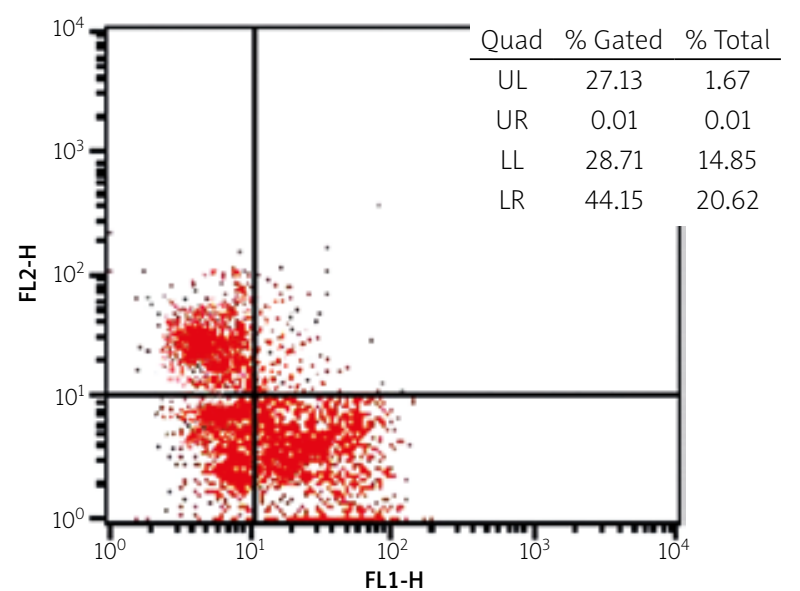

Fig. 3. Number of double-labelled CD34+CD133+ cells in peripheral blood showed in dotplot 3 hours after the injury in experimental group.

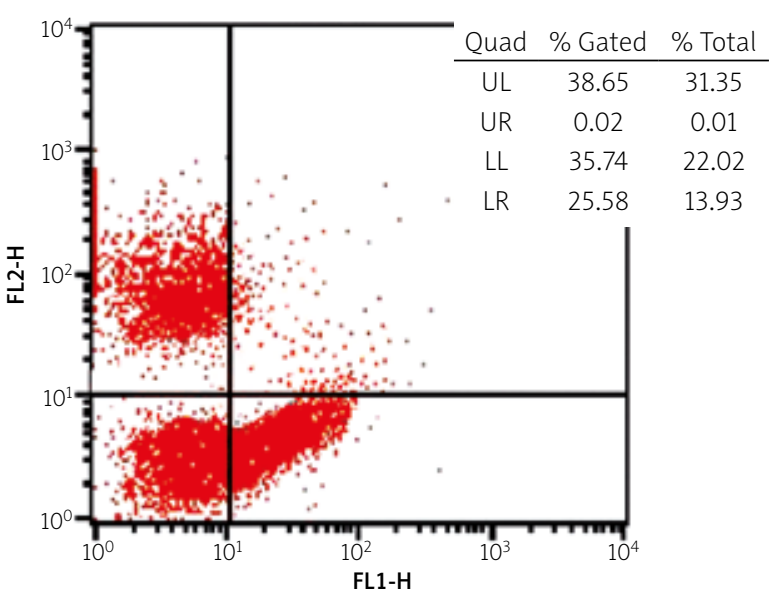

Fig. 4. Number of double-labelled CD34+CD133+ cells in peripheral blood showed in dotplot 24 hours after the injury in experimental group, indicated the number was 2 times higher than 3 hours after the injury.

Table II. Number of CD34+CD133+ cells in the peripheral blood of each group at each time point (cell/20 million mononuclear cells, $n=12$ )

\begin{tabular}{|lccccccc|}
\hline Group & $0 \mathrm{~h}$ & $3 \mathrm{~h}$ & $6 \mathrm{~h}$ & $24 \mathrm{~h}$ & $3 \mathrm{~d}$ & $7 \mathrm{~d}$ & $14 \mathrm{~d}$ \\
\hline Control group & $47 \pm 3.03$ & $22 \pm 1.38$ & $60 \pm 3.00$ & $51 \pm 3.12$ & $44 \pm 3.22$ & $46 \pm 3.82$ & $45 \pm 1.29$ \\
\hline Experimental group & $46 \pm 2.43$ & $25 \pm 0.90$ & $69 \pm 2.75^{*}$ & $71 \pm 5.33^{*}$ & $49 \pm 2.51$ & $44 \pm 2.11$ & $46 \pm 1.57$ \\
\hline
\end{tabular}
${ }^{*} p<0.05$.

The two groups exhibited minor positive expression of NSE before surgery, but NSE expression first decreased at postoperative $6 \mathrm{~h}$ and then increased at postoperative three days, which peaked on postoperative D7 and began decreasing on postoperative D14. There were significant differences in the expression of NSE between the EG and CG groups on postoperative D3 and D7 ( $p<0.05$, Table III).

In the normal group, CD31 expression was very low in the normal group (Fig. 5A), which was increased in the experiment group seven days after surgery (Fig. 5B) and decreased significantly by 14 days after surgery (Fig. 5C); expression was high in the control group seven days after surgery (Fig. 5D) and decreased by 14 days after surgery (Fig. 5E).

The expression of NSE in the normal group, shown in Figure 6A, was decreased at $6 \mathrm{~h}$ after injury (Fig. 6B) and increased seven days after injury (Fig. 6C). Expression was even higher 14 days after injury (Fig. 6D) in the experimental group, and the neurons and cytoplasm were nearly normal with a clear nucleolus.
NSE was decreased $6 \mathrm{~h}$ after injury (Fig. 6E) and increased seven days after injury (Fig. 6F). Expression was even higher 14 days after injury (Fig. 6G) in the control group. Some neurons showed atrophy with reduced cytoplasm and an unclear nucleolus.

\section{Microvessel identification and density peri-injury brain tissue}

There was less cortical microvasculature in normal rats, and MVD was increased in other groups compared to the normal group, showing significant differences $(p<0.05)$; at three and seven days after surgery, MVD of experimental rats was significantly higher than in the control group (Table IV).

\section{BrdU-positive cells in the experiment group peri-injury brain tissue}

Multiple BrdU-positive cells (transplanted cells) are shown in Figure 7. Figures 7A and B shows the cortical regions; BrdU-positive cells were neuron (arrow) 

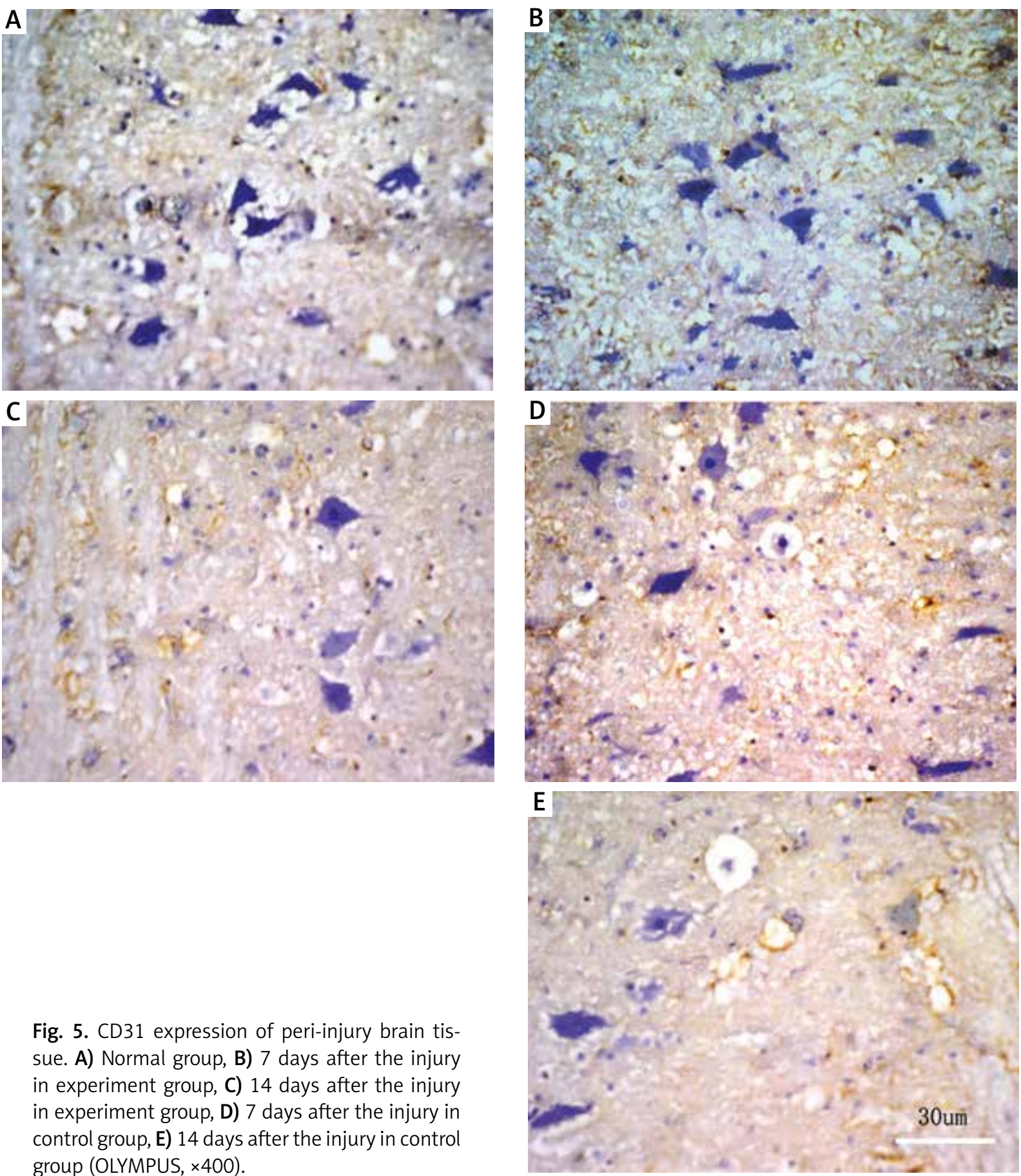

Fig. 5. CD31 expression of peri-injury brain tissue. A) Normal group, B) 7 days after the injury in experiment group, C) 14 days after the injury in experiment group, D) 7 days after the injury in control group, E) 14 days after the injury in control group (OLYMPUS, ×400).

Table III. Expression of CD31 and neuron-specific enolase (NSE) in peripheral tissues of brain $(n=20)$

\begin{tabular}{|llcccccc|}
\hline Index & \multicolumn{1}{c}{ Group } & $0 \mathrm{~h}$ & $6 \mathrm{~h}$ & $1 \mathrm{~d}$ & $3 \mathrm{~d}$ & $7 \mathrm{~d}$ & $14 \mathrm{~d}$ \\
\hline \multirow{2}{*}{ CD31 } & Control group & $1.29 \pm 0.04$ & $9.22 \pm 1.02$ & $19.87 \pm 5.03$ & $27.89 \pm 2.33$ & $31.23 \pm 2.38$ & $6.02 \pm 2.00$ \\
\cline { 2 - 8 } & Experimental group & $0.87 \pm 0.06$ & $12.98 \pm 1.11$ & $29.77 \pm 6.29^{*}$ & $34.45 \pm 3.02^{*}$ & $40.10 \pm 3.00^{*}$ & $7.30 \pm 1.19$ \\
\hline \multirow{2}{*}{ NSE } & Control group & $21.34 \pm 3.42$ & $14.92 \pm 3.87$ & $28.77 \pm 2.21$ & $31.67 \pm 3.99$ & $30.03 \pm 4.88$ & $22.22 \pm 2.01$ \\
\cline { 2 - 8 } & Experimental group & $19.88 \pm 2.77$ & $18.18 \pm 4.28$ & $32.94 \pm 3.02$ & $38.63 \pm 2.70^{*}$ & $44.29 \pm 5.29^{\star}$ & $23.20 \pm 3.40$ \\
\hline
\end{tabular}

${ }^{*} p<0.05$ 

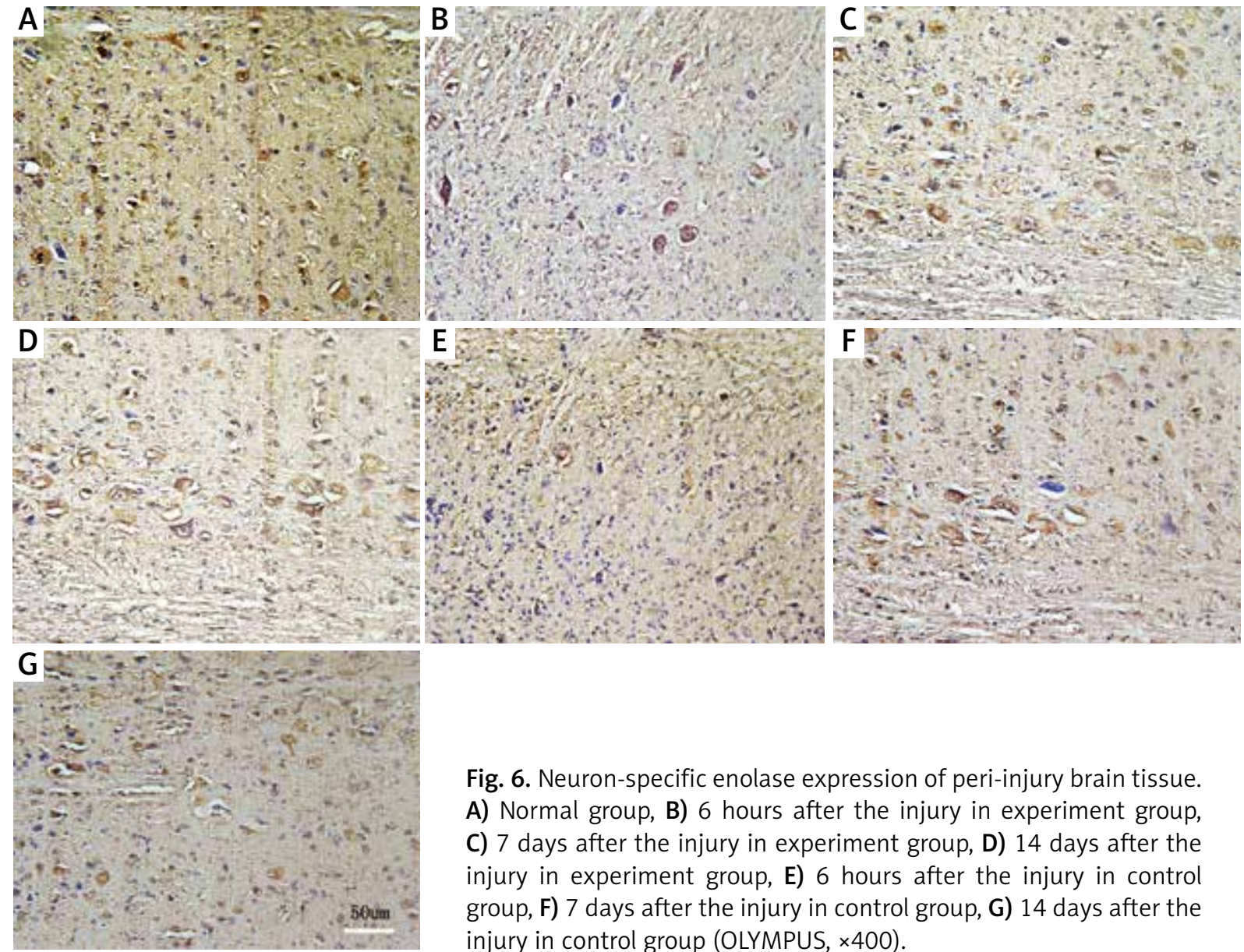

Fig. 6. Neuron-specific enolase expression of peri-injury brain tissue. A) Normal group, B) 6 hours after the injury in experiment group, C) 7 days after the injury in experiment group, D) 14 days after the injury in experiment group, E) 6 hours after the injury in control group, F) 7 days after the injury in control group, G) 14 days after the injury in control group (OLYMPUS, $\times 400$ ).

shaped, polygonal, had large cell bodies with a central nucleus, round, and the outline was clear with an obvious nucleolus. Some unclear nuclear shrinkage was observed. Figures $7 C$ and D shows white matter; the cells had a glial cell morphology (arrow), were round, had a small cell body, and the nucleus was large and clearly with less cytoplasm observed (OLYMPUS, X400, Tokyo, Japan).

\section{Comparison of modified neurological severity scores}

There were significant differences in mNSS between the two groups (ANOVA of repeated-measurement, $\mathrm{F}=5.997, p=0.034$ ), as well as significant differences in the intragroup comparison at different time points (ANOVA of repeated-measurement, $F=37.106, p=0.000)$. Group CG showed significantly higher mNSSs than group CG on D7, D14, and D21 after surgery ( $t$ test, $p<0.05$ ). The mNSS scores of the two groups first increased and then decreased over time, reaching a maximum on D7; the scores on D7 and D14 were significantly higher than on D3 ( $t$ test, $p<0.05$, Table $\mathrm{V}$ ).

\section{Discussion}

Craniocerebral injury is a common and frequently occurring neurological disease; although many treatment methods are available, such as surgery and drugs, many patients experience life-long disability. Therefore, researchers have examined the impact of stem cells on the recovery of neurological impairment.

Consistent with previous studies [3,15], CD90, a non-haematopoietic marker, showed high expression, confirming the purity of the cultured mesenchymal stem cells. Additionally, CD34, a haematopoietic marker, showed low expression. As shown in Figure 2, the CD90-positive rate of isolated BMSCs 

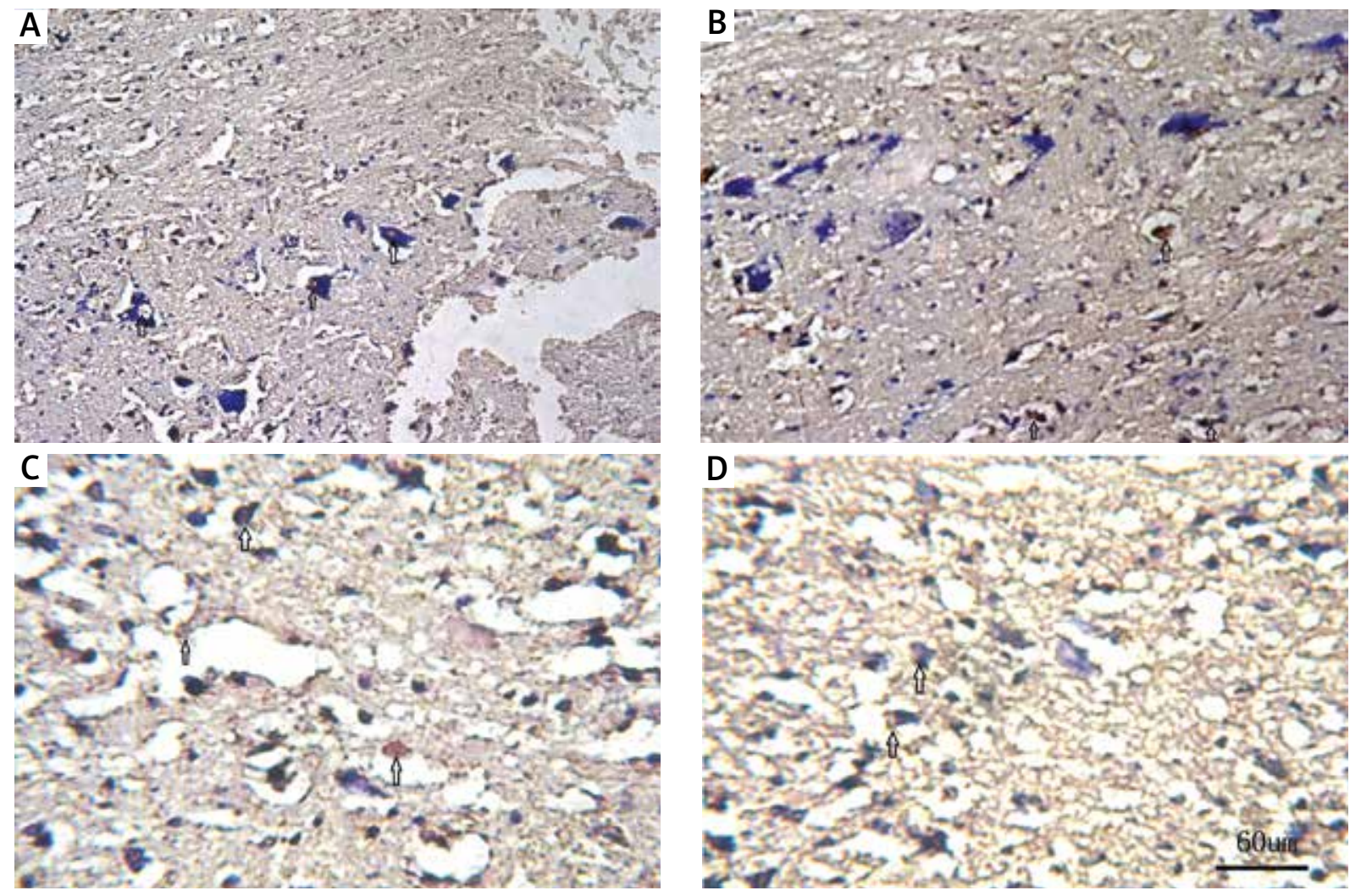

Fig. 7. BrdU-positive cells expressed in the experimental peri-injury brain tissue group. A) and B) show the cortical regions, and BrdU-positive cells were neuron (arrow) shaped, polygonal, had large cell bodies with a central nucleus, round, and the outline was clear with an obvious nucleolus. Some unclear nuclear shrinkage was observed; C) and D) show white matter, and the cells had a glial cell morphology (arrow), were round, had a small cell body, and the nucleus was large and clear with less cytoplasm observed (OLYMPUS, ×400).

was $68.49 \%$, while the CD34-positive rate was only $2.85 \%$. Additionally, as a positive marker of haematopoietic stem cells, CD34 was not primarily expressed in BMSCs. These results reflect the high purity of third-generation BMSCs.

Stem cell transplantation can help in the recovery of neurological function after $\mathrm{Cl}$. Additionally, the brain vascular system can be activated after $\mathrm{Cl}$ and begin vascular remodelling; this new vasculature is involved in the recovery of neurological function $[1,11,13,18,20]$. However, whether stem cells play a role in repairing injured nerves through neovascularisation remains unclear. This study investigated the mobilisation of CD34+CD133+ cells in the post-CI rat brain and vascular regeneration in the surrounding area by analysing neovascularisation in the surrounding area and the impact of BM-MSCs transplantation on nerve repair and functional recovery. The results showed that BM-MSC transplantation can increase the number of endothelial progenitor cells in the peripheral blood of rats with traumatic brain injury and increase the expression of peripheral vascular angiogenetic markers and neuronal markers. Neurological function in the EG group significantly improved compared to that in the CG group.

CD34 is a surface marker of haematopoietic stem cells or mature endothelial cells [6]. However, endothelial progenitor cells (EPCs) only account for a small fraction of all CD34-positive cells in the bone marrow or peripheral blood; CD133 is a surface marker of neuroepithelial stem cells [8]. With the differentiation and mature of EPCs, the expression of CD133 gradually decreased, and mature vascular endothelial cells showed no expression of CD133, making it a good molecular marker for identifying mature endothelial cells from EPCs. Thus, the number of CD34+CD133+ cells reflects the number of endo- 
Table IV. Microvessel density peri-injury brain tissue (vessels/400 field, $\bar{x} \pm \mathrm{s}$ )

\begin{tabular}{|lcccc|}
\hline Group & $0 \mathrm{~h}$ & $3 \mathrm{~d}$ & $7 \mathrm{~d}$ & $14 \mathrm{~d}$ \\
\hline Control group & $22.34 \pm 1.72$ & $53.57 \pm 2.33$ & $55.51 \pm 3.89$ & $35.53 \pm 1.73$ \\
\hline Experimental group & $23.21 \pm 1.18$ & $65.45 \pm 3.02^{*}$ & $79.38 \pm 2.00^{*}$ & $37.30 \pm 1.19$ \\
\hline
\end{tabular}

Compare with control group; ${ }^{*} p<0.05$

Table V. Comparison of modified neurological severity scores at different time points between two groups $(\bar{x} \pm s, n=6)$

\begin{tabular}{|c|c|c|c|c|c|c|c|}
\hline Group & $1 \mathrm{~d}$ & $3 d$ & $7 d$ & $14 \mathrm{~d}$ & $21 \mathrm{~d}$ & $\mathrm{~F}$ & $p$ \\
\hline Experimental group & $5.33 \pm 1.03$ & $6.00 \pm 0.89$ & $7.50 \pm 1.05^{\star}$ & $6.17 \pm 0.41^{*}$ & $4.17 \pm 0.41$ & 5.997 & 0.034 \\
\hline Control group & $5.00 \pm 0.63$ & $6.83 \pm 0.75$ & $8.17 \pm 0.75^{\text {*\# }}$ & $7.00 \pm 0.89^{* \#}$ & $5.33 \pm 0.52^{\#}$ & & \\
\hline Sum & $5.17 \pm 0.87$ & $6.42 \pm 0.90$ & $7.83 \pm 0.94$ & $6.58 \pm 0.79$ & $4.75 \pm 0.75$ & & \\
\hline
\end{tabular}

Intragroup comparison among D7, D14, and D3; ${ }^{*} p<0.05$; intergroup comparison, ${ }^{*} p<0.05$

thelial progenitor cells [14]. EPCs are precursor cells of endothelial cells and are involved in the angiogenesis and repair of endothelial cells after injury. Mobilising and transplanting EPCs can improve the angiogenetic ability of ischaemic tissues, thus promoting the repair and re-endothelialisation of damaged blood vessels. According to the results shown in Table II, Figures 3 and 4, CD34+CD133+ cells, namely EPCs, were present in the peripheral blood of rats under normal conditions, and the number of EPCs decreased rapidly in the superpermic phase ( $3 \mathrm{~h}$ after $\mathrm{Cl}$ ) but increased until higher than the normal levels by $6 \mathrm{~h}$ after $\mathrm{Cl}$; the number of EPCs in the CG group returned to normal $24 \mathrm{~h}$ after surgery, but the number of EPCs in the EG group continued to increase until $24 \mathrm{~h}$ after injury. The numbers of EPCS in the EG group 6 and $24 \mathrm{~h}$ after injury were significantly higher than that in the CG group, suggesting that BM-MSC transplantation increases the number of EPCs in the peripheral blood of $\mathrm{Cl}$ rats, with the effects persisting for approximately $24 \mathrm{~h}$.

CD31, a platelet endothelial cell adhesion molecule, is mainly expressed in endothelial cells. As an endothelial cell junction molecule, CD31 is a marker of angiogenesis in the body [9]. Table III and Figure 5 show that the expression of CD31 in the two groups was low before injury, but expression began increasing at $\mathrm{H} 6$ and on D1 after injury, was highest on D7, and began decreasing on D7. The expression of CD31 between the EG and CG groups was significantly different on D1, D3, and D7 after injury $(p<0.05)$, and the expression of CD31 from D1 to D7 was higher than in group EG, indicating that the vascular regeneration mechanism began $6 \mathrm{~h}$ after injury, reaching a peak on D7, and continuing until D14. Thus, transplanting BM-MSCs can increase the expression of angiogenetic markers around the injured area. The expression level of CD31 in peripheral brain tissue was upregulated after cerebral injury, and the subsoft subregion of corticose was relatively dense (Fig. 5), indicating that new vessels were generated from the mantle. This may be related to the wound microenvironment.

Neuron-specific enolase mainly exists in the neurons and neuroendocrine cells of the central nervous system, exhibiting a high degree of tissue specificity. As shown in Table III and Figure 6, the two groups exhibited low NSE expression before surgery; however, NSE first decreased $6 \mathrm{~h}$ after injury, but increased in both groups on $\mathrm{D} 3$, reaching a peak on D7 and decreasing on D14; the expression of NSE between the two groups showed significant differences on D3 and D7 $(p<0.05)$. Table III shows that the expressions of NSE in the two groups was normal before surgery, but decreased $6 \mathrm{~h}$ after injury, suggesting that acute neuronal injury occurred in the peripheral area. The expression of NSE in group EG increased on D3 after injury, reaching a peak on D7 and decreasing on D14, indicating that normal rats with acute brain injury strongly express NSE and use a self-repair process. The expression of NSE in the EG group was significantly higher than in group CG on D3 and D7 ( $p<0.05)$, indicating that BM-MSC transplantation increases the expression of NSE in the peri-injury area of rats with $\mathrm{Cl}$.

Figure 7 shows that the BrdU-positive cells were neuron (arrow) shaped, polygonal, had a cell body and central nucleus, were round, and had a clear outline and obvious nucleolus; in the margin white matter, most cells showed glial cell morphology (arrow), 
were round, had a small cell body and clear nuclei, as well as less cytoplasm. Combined with the regional expression of CD31 in the pia mater in cortical areas, transplanted cells underwent directed migration to the injury site and integrated the damaged region, which was coordinated with vascular regeneration, to repair the nerve. However, the mechanism of directed migration to the lesion is unknown.

Table $V$ shows that there were significant differences in mNSS between the two groups (ANOVA of repeated measurement, $F=5.997, p=0.034$ ), and mNSS in the CG group on D7, D14, and D 21 was significantly higher than in the EG group ( $t$ test, $p<0.05$ ), indicating that BM-MSC transplantation significantly improved the neurological function of $\mathrm{Cl}$ rats.

EPCs are a class of cells that have been widely examined in recent years. In 1997, Asahara et al. [2]. isolated CD34+ cells from adult peripheral blood mononuclear cells for the first time. Because these cells can differentiate into endothelial cells in vitro, express endothelial markers, and participate in angiogenesis, they were named EPCs. EPCs, also known as vascular cells, are pluripotent stem cells that can circulate, proliferate, and differentiate into precursor cells of mature vascular endothelial cells, but lack the characteristic phenotypes of mature endothelial cells.

Under ischaemic and hypoxic conditions after injury, angiogenesis and vascularisation can occur [12]. Angiogenesis refers to the process by which EPCS differentiate into endothelial cells and form the original vascular network. Vascularisation refers to the process of new capillary growth from existing blood vessels. Previous studies of adult neovascularisation consider that this process is controlled by an angiogenetic mechanism, and current studies of EPCs in the peripheral blood have shown that adult angiogenesis also includes an angiogenesis mechanism [16]. During the disease process of local tissue ischaemia or endothelial injury, EPCs can be mobilised from the bone marrow to the blood circulation, which then reach injured sites, differentiate into endothelial cells, and form new blood vessels [10,17]. Therefore, adults exhibit increased local blood flow and promoted tissue repair through the angiogenesis mechanism.

Mobilisation of EPCs after injury is very important. Under normal conditions, EPCs in the bone marrow are in a resting state and can be mobilised into the systemic circulation under many stimulating factors. An increase in the number of EPCs in peripheral circu- lation can migrate and differentiate at specific sites, thus forming endothelial cells and promoting angiogenesis. Factors that have been identified to mobilise EPCs include growth factors, such as vascular endothelial growth factor and basic fibroblast growth factor [21]. Mesenchymal stem cells (MSCs) are stem cells with self-proliferative capacity and multi-directional differentiation potential and are widely distributed in the connective tissue and organ stroma throughout the body. MSCs can be easily sampled, isolated, and largely amplified in vitro. Transplanted MSCs in vivo can secrete many vasoactive growth factors, including vascular endothelial growth factor, which is one of the main factors that promotes vascular growth [4]. MSCs can release cytokines to increase the number of EPCs in the peripheral blood circulation, which in turn promotes angiogenesis and the recovery of neurological function through angiogenesis.

The results of this study are consistent with those of previous studies. However, this study was conducted only at the molecular and behavioural levels, and thus there are some limitations. Additional studies should be conducted at the genetic level.

In conclusion, understanding the conditions of post- $\mathrm{Cl}$ neovascularisation and the impact of BM-MSCs on neovascularisation in rats with traumatic brain injury provides a foundation for $\mathrm{Cl}$ treatment by stem cell transplantation. Clinical treatment of severe traumatic brain injury is a long-term complex problem, and it is difficult to achieve breakthroughs and improvements in a short period or improve the treatment effects of patients with severe traumatic brain injury through one drug or one method. However, clinical and basic studies can improve the treatment effects of traumatic brain injury.

\section{Disclosure}

The authors report no conflict of interest.

\section{References}

1. Andrews AM, Lutton EM, Merkel SF, Razmpour R, Ramirez SH. Mechanical Injury induces brain endothelial-derived microvesicle release: implications for cerebral vascular injury during traumatic brain injury. Front Cell Neurosci 2016; 10: 43.

2. Asahara T, Murohara T, Sullivan A, Silver M, van der Zee R, Li T, Witzenbichler B, Schatteman G, Isner JM. Isolation of putative progenitor endothelial cells for angiogenesis. Science 1997; 275: 964-967.

3. Bobis S, Jarocha D, Majka M. Mesenchymal stem cells: characteristics and clinical applications. Folia Histochem Cytobiol 2006; 44: 215-230. 
4. Braghirolli DI, Helfer VE, Chagastelles PC, Dalberto TP, Gamba D, Pranke P. Electrospun scaffolds functionalized with heparin and vascular endothelial growth factor increase the proliferation of endothelial progenitor cells. Biomed Mater 2017; 12: 025003.

5. Carrion B, Kong YP, Kaigler D, Putnam AJ. Bone marrow-derived mesenchymal stem cells enhance angiogenesis via their $\alpha 6 \beta 1$ integrin receptor. Exp Cell Res 2013; 319: 2964-2976.

6. Chamoto K, Gibney BC, Lee GS, Lin M, Collings-Simpson D, Voswinckel R, Konerding MA, Tsuda A, Mentzer SJ. CD34+ progenitor to endothelial cell transition in post-pneumonectomy angiogenesis. Am J Respir Cell Mol Biol 2012; 46: 283-289.

7. Hinson HE, Rowell S, Schreiber M. Clinical evidence of inflammation driving secondary brain injury: a systematic review. J Trauma Acute Care Surg 2015; 78: 184-191.

8. Kanayasu-Toyoda T, Tanaka T, Kikuchi Y, Uchida E, Matsuyama A, Yamaguchi T. Cell-surface MMP-9 protein is a novel functional marker to identify and separate proangiogenic cells from early endothelial progenitor cells derived from CD133(+) cells. Stem Cells 2016; 34: 1251-1262.

9. Kim SJ, Kim JS, Papadopoulos J, Wook Kim S, Maya M, Zhang F, He J, Fan D, Langley R, Fidler IJ. Circulating monocytes expressing CD31: implications for acute and chronic angiogenesis. Am J Pathol 2009; 174: 1972-1980.

10. Kocher AA, Schuster MD, Szaboles MJ, Takuma S, Burkhoff D, Wang J, Homma S, Edwards NM, Itescu S. Neovascularization of ischemic myocardium by human bone derived angioblasts prevents cardio myocyte apoptosis, reduce sromodeling and improves cardiac function. Nat Med 2001; 7: 430-436.

11. Krum JM, Mani N, Rosenstein JM. Rosenstein. Roles of the endogenous VEGF receptors flt-1 and flk-1 in astroglial and vascular remodeling after brain injury. Exp Neurol 2008; 212: 108-117.

12. Lapi D, Colantuoni A. Remodeling of Cerebral Microcirculation after Ischemia-Reperfusion. J Vasc Res 2015; 52: 22-31.

13. Li Z, Wang B, Kan Z, Zhang B, Yang Z, Chen J, Wang D, Wei H, Zhang JN, Jiang R. Progesterone increases circulating endothelial progenitor cells and induces neural regeneration after traumatic brain injury in aged rats. J Neurotrauma 2012; 29: 343-353.

14. Paprocka M, Krawczenko A, Kantor A, Dus D, Carreau A, Grillon C. First immortalized human CD133+CD34- endothelial progenitor cell lines. Vasc Pharmacol 2012; 56: 361.

15. Pittenger MF, Mackay AM, Beck SC, Jaiswal RK, Douglas R, Mosca JD, Moorman MA, Simonetti DW, Craig S, Marshak DR. Multilineage potential of adult human mesenchymal stem cells. Science 1999; 284: 143-147.

16. Ratajska A, Jankowska-Steifer E, Czarnowska E, Olkowski R, Gula G, Niderla-Bielińska J, Flaht-Zabost A, Jasińska A. Vasculogenesis and Its Cellular Therapeutic Applications. Cells Tissues Organs 2017; 203: 141-152.

17. Reyes M, Dudek A, Jahagirdar B, Koodie L, Marker PH, Verfaillie CM. Origin of endothelial progenitors in human postnatal bone marrow. J Clin Invest 2002; 109: 337-346.

18. Ryu S, Lee SH, Kim SU, Yoon BW. Human neural stem cells promote proliferation of endogenous neural stem cells and enhance angiogenesis in ischemic rat brain. Neural Regen Res 2016; 11: 298-304.
19. Sherman M, Liu MM, Birnbaum S, Wolf SE, Minei JP, Gatson JW. Adult obese mice suffer from chronic secondary brain injury after mild TBI. J Neuroinflammation 2016; 13: 171.

20. Tang Y, Wang J, Lin X, Wang L, Shao B, Jin K, Wang Y, Yang GY. Neural stem cell protects aged rat brain from ischemia-reperfusion injury through neurogenesis and angiogenesis. J Cereb Blood Flow Metab 2014; 34: 1138-1147.

21. Wemer N, Kosiol S, Schiegl T, Ahlers P, Walenta K, Link A, Böhm M, Nickenig G. Circulating endothelial progenitor cells and cardiovascular outcomes. N Engl J Med 2005; 353: 999 1007.

22. Yu AY, Xu QH, Hu SL, Li F, Chen YJ, Yin Y, Zhu G, Lin JK, Feng H. Characteristics of a rat model of an open craniocerebral injury at simulated high altitude. Neuroreport 2014; 25: 1272-1280.

23. Zhao JX, Guo Q, Yuan Y, Xu W, Zhao NH. Scalp Avulsion Combined with Unusual Severe Open Craniocerebral Avulsion Injury. Chin Med J (Engl) 2015; 128: 2839-2840. 